\title{
Deriving Production Rules for Incremental View Maintenance
}

\author{
Stefano Ceri * \\ Jennifer Widom \\ IBM Almaden Research Center \\ 650 Harry Road \\ San Jose, CA 95120 \\ ceri@cs.stanford.edu,widom@ibm.com
}

\begin{abstract}
It is widely recognized that production rules in database systems can be used to automatically maintain derived data such as views. However, writing a correct set of rules for efficiently maintaining a given view can be a diffcult and ad-hoc process. We provide a facility whereby a user defines a view as an SQL select expression, from which the system automatically derives set-oriented production rules that maintain a materialization of that view.

The maintenance rules are triggeted by operations on the view's base tables. Generally, the rules perform incremental maintenance: the materialized view is modified according to the sets of changes made to the base tables, which are accessible through logical tables provided by the rule language. However, for some operations substantial recomputation may be required. We give algorithms that, based on key information, perform syntactic analysis on a view definition to determine when efficient maintenance is possible.
\end{abstract}

\section{Introduction}

In relational database systems, a view is a logical table derived from one or more physical (base) tables. Views are useful for presenting different levels of abstraction or different portions of a database to different users. Typically, a view is specified as an SQL select expression. A retrieval query over a view is written as if the view were a physical table; the query's answer is logically equivalent to evaluating the view's select expression, then performing the query using the result. There are two well-known approaches to implementing views. In the first approach, views are virtual: queries over views are modified into queries over base tables [Sto75]. In the second approach, views are materalized: they are computed from the base tables and stored in the database [BLT86,KP81,SI84]. Different applications favor one or the other approach. In this paper we consider the problem of view materialization.

Production rules in database systems allow specification of data manipulation operations that are executed automatically when certain events occur or conditions are met, e.g. [DE89, MD89, SJGP90, WF90]. Clearly, production rules can be used to maintain materialized views: when base tables change, rules are triggered that modify the view. ${ }^{1}$ Writing a correct set of rules for effi-

- Permanent address: Dip. di Elettronica, Politecnico di Milano, Piazza L. Da Vinci 32, 20133 Milano, Italy

'Production rules also can be used to implement virtual views, as shown in [SJGP9D].

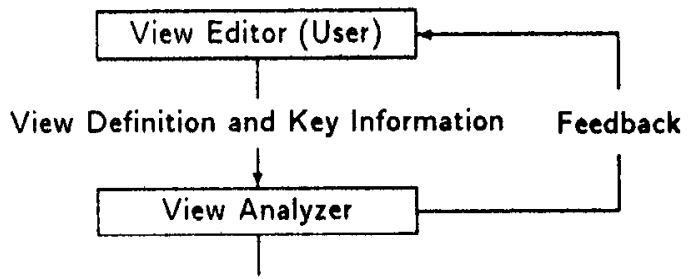

Final View with Analysis Information

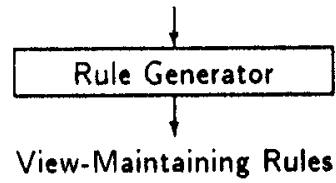

Figure 1: Rule derivation system

ciently maintaining a given view can be a difficult process, however. The rules could simply rematerialize the view from the base tables, but this can be very inefficient. Efficiency is achieved by incremental maintenance, in which the changed portions of the base tables are propagated to the view, without full recomputation. We have developed a method that automatically derives incremental maintenance rules for a wide class of views. The rules produced are executable using the rule language of the Starburst database system at the IBM Almaden Research Center [WCL91].

Figure 1 shows the structure of our system, which is invoked at compile-time when a view is created. Initially, the user enters the view as an SQL select expression, along with information about keys for the view's base tables. ${ }^{2}$ Our system then performs syntactic analysis on the view definition; this analysis determines two things: (1) whether the view may contain duplicates (2) for each base table referenced in the view, whether efficient view maintenance rules are possible for operations on that table. The user is provided with the results of this analysis. The results may indicate that, in order to improve the efficiency of view maintenance, further interaction with the system is necessary prior to rule generation. In particular:

\footnotetext{
${ }^{2}$ Key information is essential for view analysis, as we will show. Functional dependencies could be specified as well, but we assume that keys are more easily understood and specified by the user; in normalized tables, functional dependencies are captured by keys anyway.
} 
- Views with duplicates cannot be maintained efficiently, as explained in Section 4.3. Hence, if the system detects that the view may contain duplicates. then the user should add distinct to the view definition. (In SQL, distinct eliminates duplicates.)

- If the system detects that efficient maintenance rules are not possible for some base table operations, this may indicate to the user that not all key information has been included, or the user may choose to modify the view definition.

If changes are made, view analysis is repeated. In practice, we have discovered that efficient rules are possible for most views and operations once all key information is provided. However, there are cases when certain base table operations cannot be supported efficiently. If these operations are expected to occur frequently, view materialization may be inappropriate. The responsibility for considering these trade-offs lies with the user; our system provides all necessary information.

Once the user is satisfied with the view definition and its properties, the system generates the set of view-maintaining rules. Rules are produced for insert, delete, and update operations on each base table referenced in the view. The rule language we use is setoriented, meaning that rules are triggered after arbitrary sets of changes to the database (Section 3). For those operations for which the system has determined that efficiency is possible, the maintenance rules modify the view incrementally according to the changes made to the base tables. These changes are accessible using the rule system's transition table mechanism (Section 3). For those operations for which efficiency is not possible, rematerialization is performed.

Note that the view must be computed in its entirety once, after which it is maintained automatically. The frequency of view maintenance depends on the frequency of rule invocation, which is flexible; see Section 3. Our method is directly applicable for simultaneous maintenance of multiple views; see Section 9 .

\subsection{Related Work}

Most other work in incremental view maintenance differs from ours in two ways: (1) It takes an algebraic approach, considering a restricted class of views and operations. In contrast, we consider a practical class of views specified using a standard query language, and we consider arbitrary database operations. (2) It sug. gests view maintenance mechanisms that must be brilt in to the database system. In contrast, we propose view maintenance as an application of an existing mechanism. In addition, our system provides interaction whereby the user can modify a view so the system will guarantee efficient maintenance.

In [BLT80], views are specified as relational algebra expressions. Algorithms are given for determining whell base table changes are irrelevant to the view and for differentially reevaluating a view after a set of insert. and delete operations. [Han87] extends this work to exploit common subexpressions and proposes an alter- native approach using RETE networks; [Han87] also includes algorithms for incremental aggregate maintenance. In [RCBB89], an algebra of "delta relations" is described, including a "changes" operator that can be applied to views. There is a suggested connection to the production rules of HiPAC [MD89], but rule derivation is not included. In [SP89], incremental maintenance of single-table views is considered, with emphasis on issues of distribution.

Our work here is loosely related to that reported in [CW90], where we gave a method for deriving production rules that maintain integrity constraints. Our solutions to the two problems differ considerably, but the approaches are similar: In both cases we describe a general . ompile-time facility in which the user provides a high-level declarative specification, then the system uses syntactic analysis to produce a set of lower-level production rules with certain properties relative to the user's specification.

\subsection{Outline}

Section 2 defines our SQL-based syntax for view definition and Section 3 provides an overview of our production rule language. Section 4 motivates our approach: it gives an informal overview of view analysis, explains incremental maintenance, and describes certain difficulties encountered with duplicates and updates. ${ }^{8}$ Subsequent sections contain the core technical material, formally describing our methods for view analysis and rule generation. We consider top-level table references in Section 5, positively nested subqueries in Section 6, negatively nested subqueries in Section 7, and set operators in Section 8. In each of these sections we describe how view analysis can guarantee certain properties, and we show how these properties are used to determine if efficient maintenance is possible. Section 9 addresses system execution, showing that the generated rules behave correctly at run-time. Finally, in Section 10 we conclude and discuss future work.

Due to space constraints, some details have been omitted. For further details and additional examples see [CW91].

\section{View Definition Language}

Views are defined using a subset of the SQL syntax for select expressions. The grammar is given in Figure 2 and shonld be self-explanatory to readers familiar with SQI [IBM88]. ${ }^{4}$ Several examples are given in subsequent sections. Our view definition language is quite powerful, but, for brevity and to make our approach more preble, the language does include certain restrictions:

\footnotetext{
${ }^{3}$ Note that we are not dealing with the view update prob. lem, which addresses how updates on views are propagated to updates on base tables. We are considering how updates on base tables are propagated to updates on views.

${ }^{4}$ We include multi-column in (grammar productions 12 and 16), which is not standard in all SQL implementations.
} 


\begin{tabular}{|c|c|c|c|}
\hline 1. & View.Def & $::=$ & $\begin{array}{l}\text { deflne view } V(\text { Col-List }) \text { : } \\
\text { View-Exp }\end{array}$ \\
\hline 2. & View-Exp & $::=$ & Select-Exp $\mid$ Set-Exp \\
\hline 3. & Select-Exp & $::=$ & $\begin{array}{l}\text { select [distinct] Col.List } \\
\text { from Table-List } \\
\text { [where Predicate] }\end{array}$ \\
\hline 4. & Set-Exp & $::=$ & 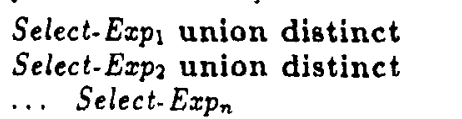 \\
\hline 5. & & 1 & 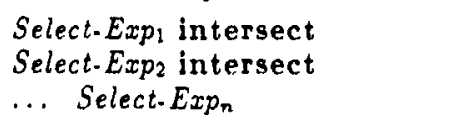 \\
\hline 6. & Col-List & $::=$ & Coll $_{1}, \ldots$, Col $_{n} \mid *$ \\
\hline 7. & Col & $::=$ & {$[T] C \mid.[$ Var. $] C$} \\
\hline 8. & Table-List & $::=$ & $T_{1}\left[\operatorname{Var}_{1}\right], \ldots, T_{n}\left[\operatorname{Var}_{n}\right]$ \\
\hline 9. & Predicate & $::=$ & Item Comp Item \\
\hline 10. & & 1 & exists (Simple-Select) \\
\hline 11. & & 1 & not exists (Simple-Select) \\
\hline 12. & & 1 & Item in (Simple-Select) \\
\hline 13. & & | & Item not in (Simple-Select) \\
\hline 14. & & 1 & Item Comp any (Simple-Select) \\
\hline 15. & & | & Predicate and Predicate \\
\hline 16. & Item & $::=$ & Col $\mid\langle$ Col-List $\rangle \mid$ constant \\
\hline 17. & Comp & $::=$ & $=|\langle|\langle\neq \mid\rangle|\rangle=| !=$ \\
\hline 18. & Simple-Select & $::=$ & $\begin{array}{l}\text { select Col-List from Table-List } \\
\text { [where Simple-Pred] }\end{array}$ \\
\hline 19. & Simple-Pred & $::=$ & Item Comp Item \\
\hline 20. & & 1 & Simple-Pred and Simple.Pred \\
\hline
\end{tabular}

Figure 2: Grammar for View Definitions

- Disjunction in predicates is omitted. (There is little loss of expressive power since or usually can be simulated using union.)

- Subqueries are limited to one level of nesting.

- Set operators union and intersect may not be mixed; set operator minus is omitted.

- Comparison operators using all are omitted.

The reader will see that our method could certainly be extended to eliminate these restrictions, but the details are lengthy. Note also that we have omitted aggregates. Incremental methods for maintaining aggregates have been presented elsewhere [Han 87 ]; these techniques can be adapted for our framework.

\section{Production Rule Language}

We provide a brief but self-contained overview of the set-oriented, SQL-based production rule language used in the remainder of the paper. Further details and numerous examples appear in [WF90, WCL91]. Here we describe only the subset of the rule language used by the view maintenance rules.

Our rule facility is fully integrated into the Starburst. database system. Hence, all the usual database functionality is available; in addition, a set of rules may be defined. Rules are based on the notion of transitions, which are database state changes resulting from execution of a sequence of data manipulation operations. We consider only the net effect of transitions, as in [BLT86, WF90]. The syntax for defining production rules is: 5

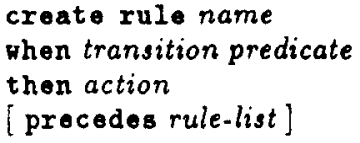

Transition predicates specify one or more operations on tables: inserted into $T$, deleted from $T$, or updated $\mathbf{T}$. A rule is triggered by a given transition if at least one of the specified operations occurred in the net effect of the transition. The action part of a rule specifies an arbitrary sequence of SQL data manipulation operations to be executed when the rule is triggered. The optional precedes clause is used to induce a partial ordering on the set of defined rules. If a rule $R_{1}$ specifies $R_{2}$ in its precedes list, then $R_{1}$ is higher than $R_{2}$ in the ordering. When no ordering is specified between two rules, their order is arbitrary but deterministic [ACL91].

A rule's action may refer to the current state of the database through top-level or nested SQL select operations. In addition, rule actions may refer to transition tables. A transition table is a logical table reflecting changes that have occurred during a transition. At the end of a given transition, transition table "inserted T" refers to those tuples of table $T$ in the current state that were inserted by the transition, transition table "deleted $\mathbf{T}$ " refers to those tuples of table $\mathbf{T}$ in the pre-transition state that were deleted by the transition, transition table "old updated $\mathbf{T}$ " refers to those tuples of table $\mathbf{T}$ in the pre-transition state that were updated by the transition, and transition table "new updated $\mathbf{T}$ " refers to the current values of the same tuples. Transition tables may be referenced in place of tables in the from clauses of select operations.

Rules are activated at rule assertion points. There is an assertion point at the end of each transaction, and there may be additional user-specified assertion points within a transaction. ${ }^{B}$ We describe the semantics of rule execution at an arbitrary assertion point. The state change resulting from the user-generated database operations executed since the last assertion point (or start of the transaction) create the first relevant transition, and some set of rules are triggered by this transition. A triggered rule $R$ is chosen from this set such that no other triggered rule is higher in the ordering. $R$ 's action is executed. After execution of $R$ 's action, all other rules are triggered only if their transition predicate holds with respect to the composite transition created by the initial transaction and subsequent execution of $R$ 's action. That is, these rules consider $R$ 's action as if it were executed as part of the initial transition. Rule $R$, however,

\footnotetext{
${ }^{5}$ Rules also may contain conditions in if clauses, but these are not needed for view maintenance.

${ }^{6}$ Currently, assertion points are at transaction commit only. We will soon extend the system with a flexible mechanisin that supports additional points [WCL91].
} 
has already "processed" the initial transition; thus, $R$ is triggered again only if its transition predicate holds with respect to the transition created by its action. From the new set of triggered rules, a rule is chosen such that no other triggered rule is higher in the ordering, and its action is executed. At an arbitrary time in rule processing, a given rule is triggered if its transition predicate holds with respect to the (composite) transition since the last time at which its action was executed; if its action has not yet been executed, it is considered with respect to the transition since the last rule assertion point or start of the transaction. When the set of triggered rules is empty, rule processing terminates.

For view maintenance, it sometimes is necessary for a rule to consider the entire pre-transition value of a table (see, e.g., Section 5.4). Currently there is no direct mechanism in the rule language for obtaining this value, but it can be derived from transition tables. In the action part of view maintenance rules, we use "old $T$ " to refer to the value of table $\boldsymbol{T}$ at the start of the transition triggering the rule. old $\mathbf{T}$ is translated to:

( $T$ minus inserted $T$ minus now updated $T$ ) union deloted $T$ union old updated $T$

This expression may seem rather complex, but one should observe that in most cases the transition tables are small or empty.

\section{Motivation}

\subsection{View Analysis}

Initially, the user defines a view using the language of Section 2, and the user specifies a set of (single- or multicolumn) keys for the view's base tables. All known keys for each table should be specified, since this provides important information for view analysis. Using the key information, during view analysis the system considers each list of table references in the view definition. For each list, it first computes the "bound columns" of the table references. Based on the bound columns, it then determines for each table reference whether the reference is "safe". When a table reference is safe, incremental view maintenance rules can be generated for operations on that table, as described in Section 4.2. The system also uses the bound columns for the top-level tables to determine if the view may contain duplicates. Formal definitions for bound columns and safety are based on the context of table references and are given in Sections $5-7$.

\subsection{Incremental Maintenance}

The definition of a view $V$ can be interpreted as an expression mapping base tables to table $V$. That is, $V=V_{\exp }\left(T_{1}, \ldots, T_{n}\right)$, where $T_{1}, \ldots, T_{n}$ are the base tables appearing in $V$ 's definition. Efficient maintenance of $V$ is achieved when changes to $T_{1}, \ldots, T_{n}$ can be propagated incrementally to $V$, without substantial recomputation. Consider any table reference $T_{i}$ in $V$, and assume for the moment that $T_{i}$ appears only once in $V$ 's definition. If view analysis determines that $T_{i}$ is safe, then changes to $T_{i}$ can be propagated incrementally to $V$. More formally, changes to $T_{i}$ (sets of insertions, deletions, or updates), denoted $\Delta T_{i}$, produce changes to $V$, denoted $\Delta V$, that can be computed using only $\Delta T_{i}$ and the other base tables: $\Delta V=V_{e x p}^{\prime}\left(T_{1}, \ldots, \Delta T_{i}, . ., T_{n}\right)$, where $V_{e x p}^{\prime}$ is an expression derived from $V_{\text {exp }}$. Table $V$ is then modified by inserting or deleting tuples from $\Delta V$ as appropriate. We assume that $\Delta T_{i}$ is small with respect to $T_{i}$ and $\Delta V$ is small with respect to $V$; hence, safe table references result in efficient maintenance rules. If $T_{i}$ appears more than once in $V$ 's definition, we separately analyze each reference. If all references are safe, then changes to $T_{i}$ can be propagated incrementally to $V$. If any reference is unsafe, changes to $T_{i}$ may cause rematerialization.

\subsection{Duplicates}

Our method does not support efficient maintenance of views with duplicates. The main difficulty lies in generating rule actions in SQL that can manipulate exact numbers of duplicates. As an example, the SQL delete operation is based on truth of a predicate; hence, if a table contains four copies of a tuple (say), there is no SQL operation that can delete exactly two copies. To correctly maintain views with duplicates, such partial deletions can be necessary. [BLT86] also considers the problem of duplicates in views, proposing two solutions. In the first solution, an extra column is added in the view table to count the number of occurrences of each tuple. We choose not to use this approach because rule generation can become quite complex and the result is not transparent to the user. (The user must reference duplicates in the view through the extra column.) The second solution proposed in [BLT86] ensures that a view will not contain duplicates by requiring it to include key columns for each of the base tables. We have essentially taken this approach, however we have devised algorithms that allow us to loosen the key requirement considerably, yet still guarantee that a view will not contain duplicates.

\subsection{Update Operations}

When update operations are performed on a view's base tables, we would like to consequently perform an update operation on the view. In many cases, however, this is not the semantic effect. As a simple example, consider two tables $T 1(A, B)$ and $T 2(C, D)$ where $T 1$ contains tuples $(x, y),(z, y)$, and $(u, v)$, and T2 contains tuples $(x, z)$ and $(v, x)$. Consider the following view:

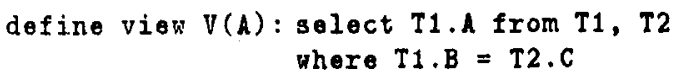

Initially, $V$ contains only one tuple, (u). Now suppose the following two update operations are performed on table T2:

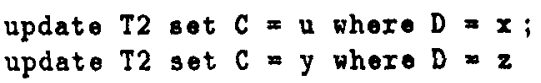

The effect of the first update is to remove tuple (u) from view $V$, while the effect of the second update is to add tuples $(x)$ and $(z)$ to $V$. There is no way to reflect the update operations on base table T2 as an update operation on view $V$; rather, the updates must be reflected as 
delete and insert operations on $V$. There do exist some cases in which update operations on base tables can be reflected as updates on views. However, for general and automatic rule derivation, in our approach update operations on base tables always result in delete and/or insert operations on the view.

\section{Top-Level Table References}

Assume now that the user has defined a view and has specified key information for the view's base tables. Assume that the view does not include set operators union or intersect; views with set operators are covered in Section 8. The system first analyzes the top-level table references, i.e., those references generated from the Table-List in grammar production 3 of Figure 2. This analysis reveals both whether the view may contain duplicates and whether efficient maintenance rules are possible for operations on the top-level tables. Consider a view $V$ with the general form: ${ }^{7}$

deflne view $V($ Col- $L$ ist $)$ : select $C_{1}, \ldots, C_{n}$ from $T_{1}, \ldots, T_{m}$ where $P$

where $T_{1}, \ldots, T_{m}$ are the top-level table references, $C_{1}, \ldots, C_{n}$ are columns of $T_{1}, \ldots, T_{m}$, and $P$ is a predicate.

\subsection{Bound Columns}

View analysis relies on the concept of bound columns. The bound columns of the top-level table references in view $V$ are denoted $B(V)$ and are computed as follows:

Definition 5.1 (Bound Columns for Top-Level Table References)

1. Initialize $B(V)$ to contain the columns $C_{1}, \ldots, C_{n}$ projected in the view definition.

2. Add to $B(V)$ all columns of $T_{1}, \ldots, T_{m}$ such that predicate $P$ includes an equality comparison between the column and a constant.

3. Repeat until $B(V)$ is unchanged:

(a) Add to $B(V)$ all columns of $T_{1}, \ldots, T_{m}$ such that predicate $P$ includes an equality comparison between the column and a column in $B(V)$.

(b) Add to $B(V)$ all columns of any table $T_{i}, 1 \leq i \leq$ $m$, if $B(V)$ includes a key for $T_{i}$.

Bound columns can be computed using syntactic analysis and guarantee the following useful property (Lemma 5.2 below): If two tuples in the cross-product of toplevel tables $T_{1}, \ldots, T_{m}$ satisfy predicate $P$ and differ in their bound columns, then the tuples also must differ in view columns $C_{1}, \ldots, C_{m}$. Let $\operatorname{Proj}\left(t, C_{1}, \ldots, C_{j}\right)$ denote the projection of a tuple $t$ onto a set of columns $C_{1}, \ldots, C_{j}$.

Lemma 5.2 (Bound Columns Lemma for TopLevel Tables) Let $t_{1}$ and $t_{2}$ be tuples in the cross. product of $T_{1}, \ldots, T_{m}$ such that $t_{1}$ and $t_{2}$ both satisfy $P$. By definition, columns $C_{1}, \ldots, C_{n}$ are in $B(V)$. If $D_{1}, \ldots, D_{k}$ are additional columns in $B(V)$ such that $t_{1}$ and $t_{2}$ are

${ }^{7}$ For clarity and without loss of generality, we omit the use of table variables here. guaranteed to differ in $C_{1}, ., C_{n}, D_{1}, . ., D_{k}$, i.e. $\operatorname{Proj}\left(t_{1}\right.$, $\left.C_{1}, \ldots, C_{n}, D_{1}, \ldots, D_{k}\right) \neq \operatorname{Proj}\left(t_{2}, C_{1}, \ldots, C_{n}, D_{1}, \ldots, D_{k}\right)$, then $t_{1}$ and $t_{2}$ also are guaranteed to differ in $C_{1}, \ldots, C_{n}$, i.e. $\operatorname{Proj}\left(t_{1}, C_{1}, \ldots, C_{n}\right) \neq \operatorname{Proj}\left(t_{2}, C_{1}, \ldots, C_{n}\right)$.

Proof: Suppose, for the sake of a contradiction, that $\operatorname{Proj}\left(t_{1}, C_{1}, \ldots, C_{n}\right)=\operatorname{Proj}\left(t_{2}, C_{1}, . ., C_{n}\right)$. Then there must be some $D_{i}$ in $D_{1}, \ldots, D_{k}$ such that $\operatorname{Proj}\left(t_{1}, D_{i}\right) \neq$ $\operatorname{Proj}\left(t_{2}, D_{i}\right)$. We show that this is impossible. Consider any column $D_{i}$ in $D_{1}, \ldots, D_{k}$. Since $D_{i}$ is in $B(V)$, by the recursive definition of $B(V)$ and since $t_{1}$ and $t_{2}$ both satisfy predicate $P$, the value of column $\dot{D}_{i}$ in both $t_{1}$ and $t_{2}$ must either

1. satisfy an equality with a constant $k$, or

2. satisfy an equality with a column $C_{j}$ in $C_{1}, \ldots, C_{n}$, or

3. be functionally dependent on a constant $k$ or column $C_{j}$. (This is the case where $D_{i}$ was added to $B(V)$ because a key for $D_{i}$ 's table was present; recall that all columns of a table are functionally dependent on any key for that table.)

In the case of a constant, $\operatorname{Proj}\left(t_{1}, D_{i}\right)$ and $\operatorname{Proj}\left(t_{2}, D_{i}\right)$ are both equal to or functionally dependent on the same constant, so $\operatorname{Proj}\left(t_{1}, D_{i}\right)=\operatorname{Proj}\left(t_{2}, D_{i}\right)$. In the case of a column $C_{j}, \operatorname{Proj}\left(t_{1}, C_{j}\right)=\operatorname{Proj}\left(t_{2}, C_{j}\right)$ by our supposition, so $\operatorname{Proj}\left(t_{1}, D_{i}\right)=\operatorname{Proj}\left(t_{2}, D_{i}\right)$.

\subsection{Duplicate Analysis}

If $V$ 's definition does not include distinct, then our system performs duplicate analysis. If this analysis reveals that $V$ may contain duplicates, then the user is notified that maintenance rules cannot be generated for $V$ unless $V$ 's definition is modified to include distinct. (The system does not add distinct automatically since it may change the view's semantics.) Once the bound columns for top-level table references have been computed, duplicate analysis is straightforward:

Theorem 5.3 (Duplicates) If $B(V)$ includes a key for every top-level table, then $V$ will not contain duplicates.

Proof: Let $t_{1}$ and $t_{2}$ be two different tuples in the cross-product of the top-level tables in $V$ such that $t_{1}$ and $t_{2}$ both satisfy predicate $P$. We must show that $t_{1}$ and $t_{2}$ cannot produce duplicate tuples in $V$, i.e. $\operatorname{Proj}\left(t_{1}, C_{1}, \ldots, C_{n}\right) \neq \operatorname{Proj}\left(t_{2}, C_{1}, . ., C_{n}\right)$. By the theorem's assumption, there must be additional columns $D_{1}, \ldots, D_{k}$ in $B(V)$ such that $C_{1}, \ldots, C_{n}, D_{1}, \ldots, D_{k}$ include a key for every top-level table. Then $t_{1}$ and $t_{2}$ must differ in $C_{1}, \ldots, C_{n}, D_{1}, \ldots, D_{k}$. Consequently, by Lemma 5.2, $\operatorname{Proj}\left(t_{1}, C_{1}, . ., C_{n}\right) \neq \operatorname{Proj}\left(t_{2}, C_{1}, . ., C_{n}\right)$.

\subsection{Safety Analysis}

Safety of top-level table references is similar to duplicate analysis:

Definition 5.4 (Safety of Top-Level Table References) Top-level table reference $T_{i}$ is safe in $V$ if $B(V)$ includes a key for $T_{i}$.

The following three theorems show that if table reference $T_{i}$ is safe, then insert, delete, and update operations on $T_{i}$ can be reflected by incremental changes to $V$. 
Theorem 5.5 (Insertion Theorem for Top-Level Tables) Let $T_{i}$ be a safe top-level table reference in $V$ and suppose a tuple $t$ is inserted into $T_{i}$. If $v$ is a tuple in the cross-product of the top-level tables using tuple $t$ from $T_{i}$, and $v$ satisfies predicate $P$ so that $\operatorname{Proj}(v$, $\left.C_{1}, \ldots, C_{n}\right)$ is in view $V$ after the insertion, then $\operatorname{Proj}(v$, $\left.C_{1}, \ldots, C_{n}\right)$ was not in $V$ before the insertion.

Proof: Suppose, for the sake of a contradiction, that there was a tuple $v^{\prime}$ in $V$ before the insertion such that $\operatorname{Proj}\left(v^{i}, C_{1}, \ldots, C_{n}\right)=\operatorname{Proj}\left(v, C_{1}, \ldots, C_{n}\right)$. Let $D_{1}, \ldots, D_{k}$ be additional bound columns so that $C_{1}, \ldots, C_{n}, D_{1}, \ldots, D_{k}$ includes a key for $T_{i}$. (We know such columns exist since $T_{i}$ is safe.) Since $v$ and $v^{\prime}$ include different tuples from $T_{i}$, then $\operatorname{Proj}\left(v, C_{1}, \ldots, C_{n}, D_{1}, \ldots, D_{k}\right) \neq \operatorname{Proj}\left(v^{\prime}\right.$, $\left.C_{1}, \ldots, C_{n}, D_{1}, \ldots, D_{k}\right)$. Hence, by Lemma 5.2, Proj $(v$, $\left.C_{1}, \ldots, C_{n}\right) \neq \operatorname{Proj}\left(v^{\prime}, C_{1}, \ldots, C_{n}\right)$.

The practical consequence of this theorem is that if a set of tuples $\Delta T_{i}$ are inserted into $T_{i}$, then the tuples $\Delta V$ that should be inserted into $V$ can be derived from the cross-product of the top-level tables using $\Delta T_{i}$ instead of $T_{i}$. This exactly corresponds to the definition of incremental maintenance in Section 4.2, and is implemented in the rules given below.

Similar theorems with similar consequences apply for delete and update operations. The proofs are omitted since they also are similar [CW91].

Theorem 5.6 (Deletion Theorem for Top-Level Tables) Let $T_{i}$ be a safe top-level table reference in $V$ and suppose a tuple $t$ is deleted from $T_{i}$. If $t$ is a tuple in the cross-product of the top-level tables using tuple $t$ from $T_{i}$, and $v$ satisfies predicate $P$ so that $\operatorname{Proj}\left(v, C_{1}, \ldots, C_{n}\right)$ was in view $V$ before the deletion, then $\operatorname{Proj}\left(v, C_{1}, \ldots, C_{n}\right)$ is not in $V$ after the deletion.

Theorem 5.7 (Update Theorem for Top-Level Tables) Let $T_{i}$ be a safe top-level table reference in $V$ and suppose a tuple $t$ is updated in $T_{i}$. Let $v_{O}$ be a tuple in the cross-product of the top-level tables using the old value of tuple $t$ from $T_{i}$, where $v_{O}$ satisfies $P$ so that $\operatorname{Proj}\left(v_{0}, C_{1}, \ldots, C_{n}\right)$ was in view $V$ before the update Let $v_{N}$ be a tuple in the cross-product of the top-level tables using the new value of tuple $t$ from $T_{i}$, where $v_{N}$ satisfies $P$ so that $\operatorname{Proj}\left(v_{N}, C_{1}, \ldots, C_{n}\right)$ is in $V$ after the update. Finally, let $v$ be a tuple in the cross-product of the top-level tables not using $t$, where $v$ satisfies $P$ so is in $V$ both before and after the update. Then $\operatorname{Proj}\left(v_{0}\right.$, $\left.C_{1}, \ldots, C_{n}\right) \neq \operatorname{Proj}\left(v, C_{1}, \ldots, C_{n}\right)$ and $\operatorname{Proj}\left(v_{N}, C_{1}, \ldots, C_{n}\right)$ $\neq \operatorname{Proj}\left(v, C_{1}, \ldots, C_{n}\right)$.

\subsection{Rule Generation}

We describe how maintenance rules are generated for the top-level tables. We first consider safe table references. then unsafe references. Initially, for each table reference we generate four rules-one triggered by inserted, one by deleted, and two by updated. Subsequently we explain how some rules can be combined and how the entire rule set is ordered.

Let $T_{i}$ be a safe top-level table reference in view $V$ defined as above. If tuples are inserted into $T_{i}$, then we want to insert into $V$ those tuples produced by the view definition using inserted $\mathbf{T i}$ instead of $\mathbf{T i}$ in the toplevel table list. By Theorem 5.5, these insertions cannot create duplicates in the view. However, if a similar rule is applied because tuples also were inserted into a different top-level table, then duplicates could appear. Hence, before inserting a new tuple, the rule must ensure that the tuple has not already been inserted by a different rule. This is checked efficiently using transition table inserted $\mathrm{V}$. The rule for inserted is:

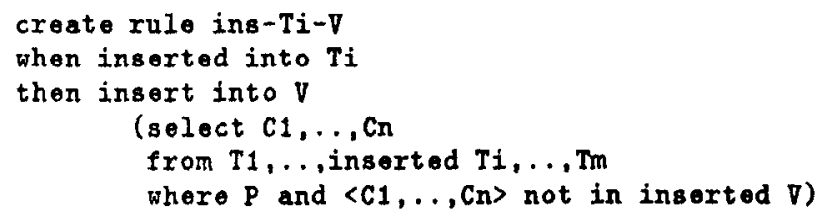

If tuples are deleted from $T_{i}$, then we want to delete from $V$ those tuples produced by the view definition using deleted $\mathbf{T i}$ instead of $\mathbf{T i}$ in the top-level table list. By Theorem 5.6, we know that these tuples should no longer be in the view. Again, however, we must remember that other tables in the top-level table list may have been modified. Hence, to identify the correct tuples to delete from $V$, we must consider the pre-transition value of all other tables, obtained using the old feature described in Section 3. For predicate $P$, let $P$-old denote $P$ with all table references $\mathbf{T}$ replaced by old $\mathbf{T}$. The rule for deleted is:

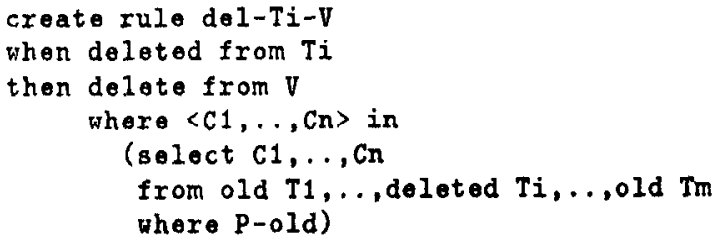

As explained in Section 4.4, update operations on base tables always cause delete and/or insert operations on views. In fact, we generate two separate rules triggered by updated-one to perform deletions and the other to perform insertions. They are similar to the rules for deleted and inserted, and their correctness follows from Theorem 5.7:



If a table appears more than once in the top-level table list, then rules are generated for each reference. Rules with identical triggering operations whose actions perform the same operation (either insert or delete) are 
create rule ner-upd-res-special-meals

whon updated ros

then insert into special-meals

(select res.seat, psgr.meal

Irom new updated res, psgr

where res.flight-id = FID

and res.psgr-id = psgr.p8gr-id

and pogr.meal != nuII

and <seat, moal> not in

inserted special-meals)

As a second example, consider the following view, which provides the frequent flier numbers of all passengers currently holding reservations:

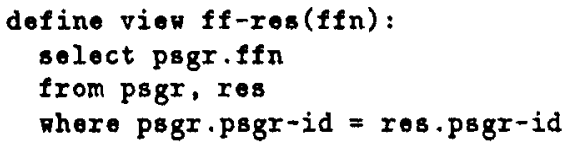

The bound columns are all columns of table psgr (since ff $n$ is a key) and column res.psgr-id. Since the bound columns do not include a key for table res, the view may contain duplicates, and distinct must be added. Table reference psgr is safe, so the rules for operations on psgr are similar to those in the previous example. Table reference res is unsafe, however, so the following rules are generated:

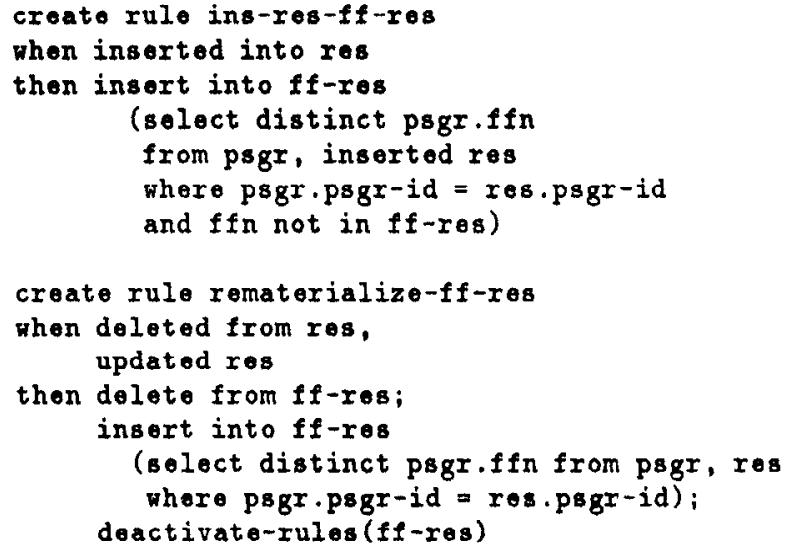

\section{Positively Nested Subqueries}

A positively nested subquery is a nested select expression preceded by exists, in, or Comp any, where Comp is any comparison operator except $!=$. We first describe safety analysis and rule generation for table references in exists subqueries. Similar methods apply for the other positively nested subqueries and are explained in Section 6.3. Consider a view $V$ as follows, where $N_{1}, \ldots, N_{l}$ are the table references under consideration:

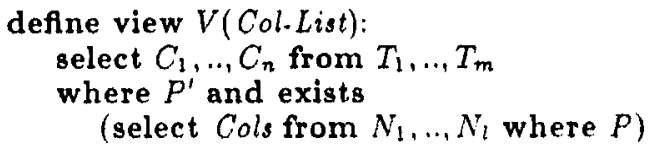

\subsection{Bound Columns and Safety Analysis}

To analyze nested table references we introduce the concept of columns that are bound by correlation to the bound columns of the top-level tables. We assume that set $B(V)$ of top-level bound columns already has been computed. Correlated bound columns are denoted $C(V)$, and for exists they are computed as follows:

Definition 6.1 (Correlated Bound Columns for Exists)

1. Initialize $C(V)$ to contain all columns of $N_{1}, \ldots, N_{l}$ such that predicate $P$ includes an equality comparison between the column and a column in $B(V)$.

2. Add to $C(V)$ all columns of $N_{1}, \ldots, N_{l}$ such that predicate $P$ includes an equality comparison between the column and a constant.

3. Repeat until $C(V)$ is unchanged:

(a) Add to $C(V)$ all columns of $N_{1}, \ldots, N_{l}$ such that predicate $P$ includes an equality comparison between the column and a column in $C(V)$.

(b) Add to $C(V)$ all columns of any table $N_{i}, 1 \leq i \leq$ $l$, if $C(V)$ includes a key for $N_{i}$.

Correlated bound columns for exists guarantee the following property:

Lemma 6.2 (Bound Columns Lemma for Exists) Consider four tuples, $t_{1}$ and $t_{2}$ in the cross-product of $T_{1}, \ldots, T_{m}$ and $n_{1}$ and $n_{2}$ in the cross-product of $N_{1}, \ldots, N_{l}$, such that $t_{1}$ and $t_{2}$ satisfy predicate $P^{\prime}, n_{1}$ satisfies nested predicate $P$ using $t_{1}$ for the top-level crossproduct, and $n_{2}$ satisfies $P$ using $t_{2}$ for the top-level cross-product. Let $D_{1}, \ldots, D_{k}$ be columns of $N_{1}, \ldots, N_{l}$ in $C(V)$ such that $n_{1}$ and $n_{2}$ are guaranteed to differ in $D_{1}, \ldots, D_{k}$, i.e. $\operatorname{Proj}\left(n_{1}, D_{1}, . ., D_{k}\right) \neq \operatorname{Proj}\left(n_{2}\right.$, $\left.D_{1}, \ldots, D_{k}\right)$. Then $t_{1}$ and $t_{2}$ are guaranteed to differ in $C_{1}, \ldots, C_{n}$, i.e. $\operatorname{Proj}\left(t_{1}, C_{1}, \ldots, C_{n}\right) \neq \operatorname{Proj}\left(t_{2}, C_{1}, \ldots, C_{n}\right)$.

Proof: Suppose, for the sake of a contradiction, that $\operatorname{Proj}\left(t_{1}, C_{1}, \ldots, C_{n}\right)=\operatorname{Proj}\left(t_{2}, C_{1}, . ., C_{n}\right)$. By supposition there is some $D_{i}$ in $D_{1}, . ., D_{k}$ such that $\operatorname{Proj}\left(n_{1}\right.$, $\left.D_{i}\right) \neq \operatorname{Proj}\left(n_{2}, D_{i}\right) . D_{i}$ is in $C(V)$, so by the recursive definitions of $C(V)$ and $B(V)$, since $t_{1}$ and $t_{2}$ satisfy $P^{\prime}$, and since $n_{1}$ with $t_{1}$ and $n_{2}$ with $t_{2}$ both satisfy predicate $P$, the value of column $D_{i}$ in both $n_{1}$ and $n_{2}$ must either

1. satisfy an equality with a constant $k$, or

2. satisfy an equality with a column $C_{j}$ in $C_{1}, \ldots, C_{n}$, or

3. be functionally dependent on a constant $k$ or column $C_{j}$.

As in Bound Columns Lemma 5.2, in all cases $\operatorname{Proj}\left(n_{1}\right.$, $\left.D_{i}\right)=\operatorname{Proj}\left(n_{2}, D_{i}\right)$

Safety analysis and rule generation for positively nested subqueries is similar to top-level tables:

Definition 6.3 (Safety of Table References for Exists ) Table reference $N_{i}$ in an exists subquery is safe in $V$ if $C(V)$ includes a key for $N_{i}$.

The following three theorems show that if $N_{i}$ is safe, then insert, delete, and update operations on $N_{i}$ can be reflected by incremental changes to $V$. We include a proof for the insertion theorem only; the other proofs follow by analogy. 
Theorem 6.4 (Insertion Theorem for Exists) Let $N_{i}$ be a safe table reference in an exists subquery in $V$ and suppose a tuple $n_{i}$ is inserted into $N_{i}$. Let $v$ be a tuple in the cross-product of the top-level tables such that $v$ satisfies $P^{\prime}$ and there is a tuple $n$ in the cross-product of the nested tables using $n_{i}$ such that $n$ satisfies $P$ using $v$, so $\operatorname{Proj}\left(v, C_{1}, \ldots, C_{n}\right)$ is in view $V$ after the insertion. Then $\operatorname{Proj}\left(v, C_{1}, \ldots, C_{n}\right)$ was not in $V$ before the insertion.

Proof: Suppose, for the sake of a contradiction, that $\operatorname{Proj}\left(v, C_{1}, \ldots, C_{n}\right)$ was in $V$ before the insertion. Then there must have been a tuple $n^{\prime}$ in the cross-product of the nested tables before the insertion and a tuple $v^{\prime}$ in the top-level cross-product such that $\operatorname{Proj}\left(v^{\prime}, C_{1}, \ldots, C_{n}\right)$ $=\operatorname{Proj}\left(v, C_{1}, \ldots, C_{n}\right), v^{\prime}$ satisfies $P^{\prime}$, and $n^{\prime}$ satisfies $P$ using $v^{\prime}$. Let $D_{1}, \ldots, D_{k}$ be correlated bound columns of $N_{1}, \ldots, N_{l}$ such that $D_{1}, \ldots, D_{k}$ includes a key for $N_{i}$. Since $n$ and $n^{\prime}$ use different tuples from $N_{i}, \operatorname{Proj}\left(n, D_{1}, \ldots, D_{k}\right)$ $\neq \operatorname{Proj}\left(n^{\prime}, D_{1}, \ldots, C_{k}\right)$. Then, by Lemma 6.2, Proj $\left(v^{\prime}\right.$, $\left.C_{1}, \ldots, C_{n}\right) \neq \operatorname{Proj}\left(v, C_{1}, \ldots, C_{n}\right)$.

Theorem 6.5 (Deletion Theorem for Exists) Let $N_{i}$ be a safe table reference in an exists subquery in $V$ and suppose a tuple $n_{i}$ is deleted from $N_{i}$. Let $v$ be a tuple in the cross-product of the top-level tables such that $v$ satisfies $P^{\prime}$ and there is a tuple $n$ in the cross-product of the nested tables using $n_{i}$ such that $n$ satisfies $P$ using $v$, so $\operatorname{Proj}\left(v, C_{1}, \ldots, C_{n}\right)$ was in view $V$ before the deletion. Then $\operatorname{Proj}\left(v, C_{1}, \ldots, C_{n}\right)$ is not in $V$ after the deletion.

Theorem 6.6 (Update Theorem for Exists) Let $N_{i}$ be a safe table reference in an exists subquery in $V$ and suppose a tuple $n_{i}$ is updated in $N_{i}$. Let $v_{O}$ be a tuple in the cross-product of the top-level tables such that $v_{O}$ satisfies $P^{\prime}$ and there is a tuple $n_{O}$ in the crossproduct of the nested tables using the old value of $n_{i}$ such that $n_{O}$ satisfies $P$ using $v_{O}$, so $\operatorname{Proj}\left(v_{O}, C_{1}, \ldots, C_{n}\right)$ was in view $V$ before the update. Let $v_{N}$ be a tuple in the cross-product of the top-level tables such that $v_{N}$ satisfies $p^{\prime}$ and there is a tuple $n_{N}$ in the cross-product of the nested tables using the new value of $n_{i}$ such that $n_{N}$ satisfies $P$ using $v_{N}$, so $\operatorname{Proj}\left(v_{N}, C_{1}, \ldots, C_{n}\right)$ is in $V$ after the update. If $\operatorname{Proj}\left(v_{0}, C_{1}, \ldots, C_{n}\right) \neq \operatorname{Proj}\left(v_{N}\right.$. $\left.C_{1}, \ldots, C_{n}\right)$, then $\operatorname{Proj}\left(v_{0}, C_{1}, \ldots, C_{n}\right)$ is not in $V$ after the update and $\operatorname{Proj}\left(v_{N}, C_{1}, \ldots, C_{n}\right)$ was not in $V$ before the update.

\subsection{Rule Generation}

First consider safe table references. The properties guaranteed by Theorems 6.4-6.6 allow incremental maintenance to be performed just as for safe top-level table references: $N_{i}$ is replaced by inserted $\mathrm{Ni}$ in the inserted rule, by deleted $\mathrm{Ni}$ in the deleted rule, and by old updated $\mathrm{Ni}$ and new updated $\mathrm{Ni}$ in the two updated rules. In the rules that perform insertions, we must check that tuples have not already been inserted by another rule; in the rules that perform deletions we must use the old value of other tables. If a table appears more than once in $N_{1}, \ldots, N_{l}$, or if a table in $N_{1}, \ldots, N_{l}$ also appears elsewhere in the view definition, then rules are merged as previously described. Unsafe table references also are handled similarly to top-level tables: If nested table reference $N_{i}$ is unsafe, triggering operations deleted from $\mathrm{Ni}$ and updated $\mathrm{Ni}$ are included in the distinguished rematerialization rule for $V$. The inserted rule is similar to the safe rule, except "not in V" is added to the predicate rather than "not in inserted V".

\subsection{Other Positively Nested Subqueries}

Safety analysis and rule generation for subqueries preceded by $\langle$ any, $<=$ any, >any, and $>=$ any is identical to exists. The method for = any and in (which are equivalent) also is identical to exists, except the set of correlated bound columns may be larger. Consider a view $V$ of the form:

$$
\begin{aligned}
& \text { define view } V(\text { Col-List }): \\
& \text { select } C_{1}, \ldots, C_{n} \text { from } T_{1}, \ldots, T_{m} \\
& \text { where } P^{\prime} \text { and }\left\langle D_{1}, \ldots, D_{j}\right\rangle \text { in } \\
& \quad \text { (select } E_{1}, \ldots, E, \text { from } N_{1}, \ldots, N_{l} \text { where } P \text { ) }
\end{aligned}
$$

Definition 6.1 of correlated bound columns is modified to include the case:

- Add to $C(V)$ every column $E_{i}$ such that corresponding column $D_{i}$ is in $B(V), 1 \leq i \leq j$.

The reader may note that view $V$ above is equivalent to view $V^{\prime}$;

$$
\begin{aligned}
& \text { define view } V^{\prime}(\text { Col-List): } \\
& \text { select } C_{1}, \ldots, C_{n} \text { from } T_{1}, \ldots, T_{m} \\
& \text { where } P^{\prime} \text { and exists } \\
& \text { (select } * \text { from } N_{1}, \ldots, N_{t} \text { where } P \\
& \quad \text { and } D_{1}=E_{1} \text { and } \ldots \text { and } D_{j}=E_{j} \text { ) }
\end{aligned}
$$

As expected, the correlated bound columns of view $V^{\prime}$ using Definition 6.1 for exists are equivalent to the correlated bound columns of $V$ using the extended definition for in. ${ }^{10}$

\subsection{Example}

Using the airline reservations database introduced in Section 5.5, the following view provides the ID's of all passengers with more than 50,000 frequent flier miles:

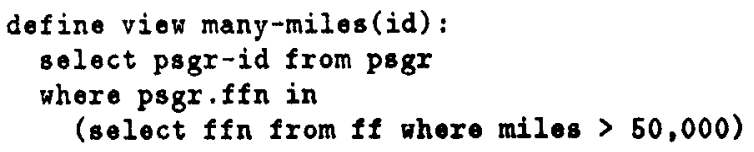

All columns of top-level table psgr are bound since psgr-id is a key. Using our extended definition for in, $\mathrm{ff}$. $\mathrm{f} \mathrm{n}$ is a correlated bound column. Since $\mathrm{ftn}$ is a key, nested table reference $f f$ is safe. The inserted and deleted rules for table $f f$ follow; the updated rules are similar.

\footnotetext{
${ }^{10}$ The reader may also note that select expressions with positive subqueries often can be transformed into equivalent select expressions without subqueries, as in [CG85, Kim82]. By considering the actual transformations, we see that the maintenance rules produced for any transformed view are equivalent to the maintenance rules produced for the original view.
} 


\subsection{Rule Generation}

If nested table reference $N_{i}$ is I-safe, then, using Theorem 7.2, the following incremental rule is generated:

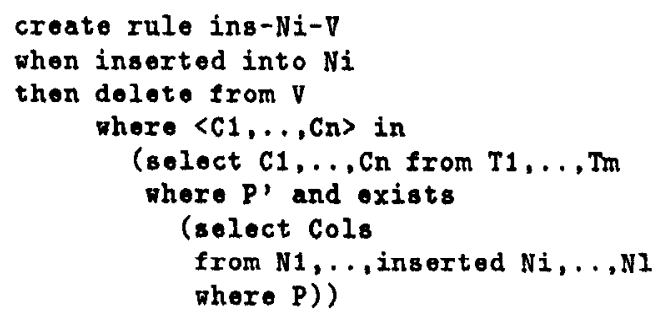

Notice that the subquery's "not exists" is converted to "exists"; this conversion occurs in the deleted and updated rules as well. If $N_{i}$ is not I-safe, then the view expression would need to be reevaluated to determine which tuples should be deleted. Hence in the unsafe case, inserted into $\mathrm{Ni}$ is included in the rematerialization rule for $V$.

If $N_{i}$ is DU-safe, then, using Theorems 7.4 and 7.5 , the following incremental rule for deleted is generated. The rules for updated correspond to the inserted and deleted rules as previously.

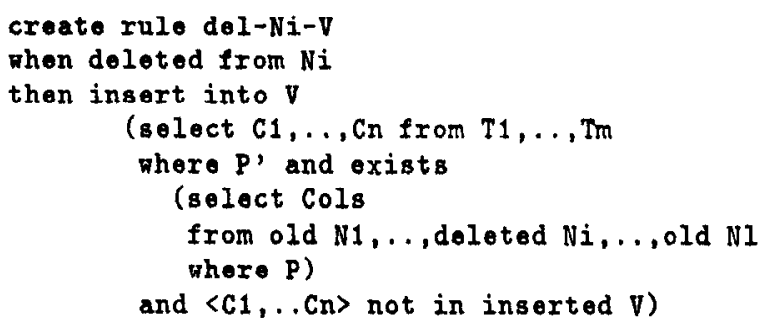

If table reference $N_{i}$ is not DU-safe, updated $\mathrm{Ti}$ is included in the rematerialization rule for $V$. For deleted, however, incremental maintenance still can be performed-as previously, for the unsafe case the rule above is modified to use "not in $V$ " rather than "not in inserted V".

\subsection{Example}

Using the airline reservations database introduced in Section 5.5, the following view provides the ID's of all reservations whose flight-id is not in table flight:

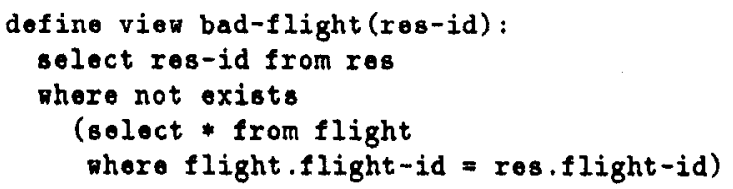

By Definitions 7.1 and 7.3 , nested table reference $\mathrm{flight}$ is both I-safe and DU-safe. The inserted and deleted rules for table flight follow; the updated rules are similar.

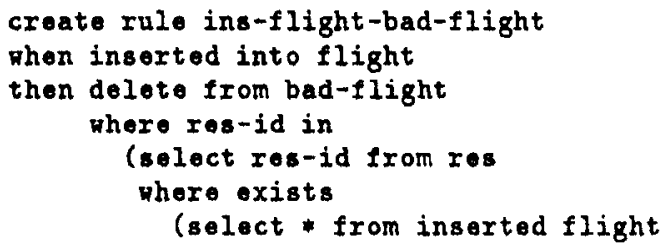

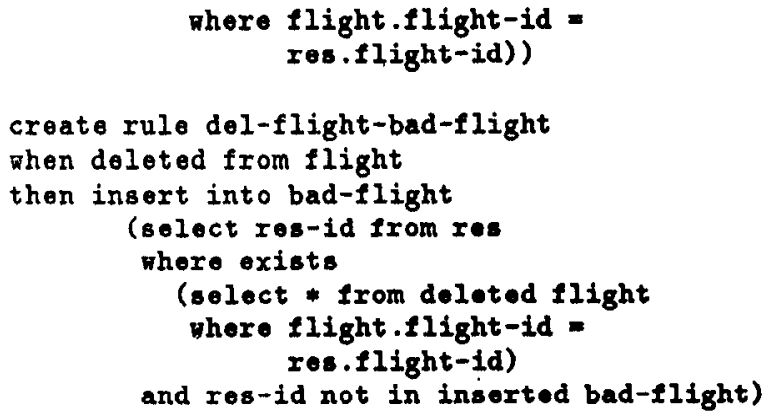

\section{Set Opcrators}

Finally, consider views with set operators. A view definition may include either union distinct or intersect. For these views, view analysis and rule generation initially is performed independently on each component select expression. The rules are then modified to incorporate the set operators.

\subsection{Union Views}

Consider a view $V$ of the form:

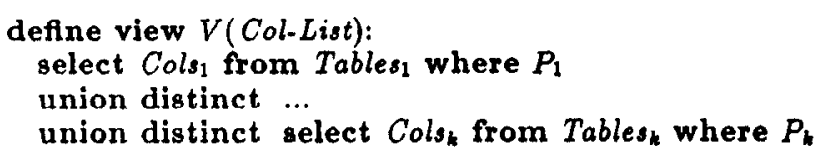

First, duplicate analysis is performed on each select expression as in Section 5.2; if any select expression may contain duplicates, the user is required to add distinct to that select expression. For each select expression, an initial set of view-maintaining rules is generated using the methods of the preceding sections. The rules' actions are then modified to incorporate union. In actions that perform insert operations, if "not in inserted V" has been added to predicate $P_{i}$ due to a safe table reference, it is changed to "not in $V$ "; this ensures that duplicates are not added by different select expressions. If the rule already includes "not in $\mathbf{~ " ~ d u e ~ t o ~ a n ~ u n s a f e ~ t a b l e ~ r e f - ~}$ erence, it remains unchanged. Modifications for delete operations are more complicated. If a tuple no longer is produced by one of the select expressions, it should be deleted from $V$ only if it is not produced by any of the other select expressions. Without loss of generality, consider a delete operation in the action of a rule generated from the first select expression in $V$. The following conjunct must be added to the delete operation's where clause:

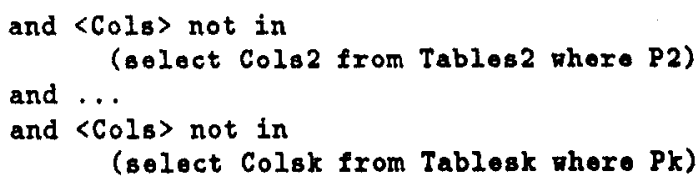

Clearly, such conjuncts may cause considerable recomputation, depending on the complexity of the select expressions. For rules in which the recomputation cost appears large, the user may choose to move the triggering operation to the rematerialization rule for $V$. 
As usual, rules with common triggering and action operations are merged, and rules whose triggering operations also appear in the rematerialization sule are eliminated.

\subsection{Intersect Views}

A view $V$ with intersect operators is handled similarly to views with union operators. In rule modification, however, all rules performing delete operations remain unchanged. (If a tuple is deleted from any select expression, then it always should be deleted from $V$.) Modifications for insert operations are similar to the modifications for delete operations in union views: If a tuple is newly produced by one of the select expressions, it should be inserted into $V$ only if it also is produced by all the other select expressions. Consider an insert operation in the action of a rule generated from the first select expression in $V$. The following conjunct must be added to the where clause of the insert operation's select expression:

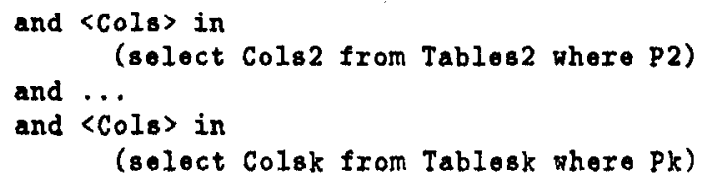

Again, if the select expressions are sufficiently complex, the user may decide that rematerialization is more appropriate.

\section{System Execution}

So far, we have described only the compile-time aspects of out facility. View definition, view analysis, and rule generation all occur prior to database system execution. We still must ensure that, at run-time, derived rules will behave as desired, i.e., views will be maintained correctly. Suppose our facility has been used to derive sets of maintenance rules for several views. The system orders the set of rules for each view so that all delete operations in rule actions precede all insert operations. No ordering is necessary between rules for different views. the action part of each rule modifies only the view itself. so rules for different views have no effect on each other.

Consider the set of rules for a given view $V$, and suppose an arbitrary set of changes has been made to $V$ 's base tables. If the rematerialization rule for $V$ is triggered, the view certainly is maintained correctly: $V$ is recomputed from its base tables; all other rules for $V$ are deactivated, so $V$ cannot be modified until the base tables change again. Suppose the rematerialization rule is not triggered. During rule processing, first some rules delete tuples from $V$, then other rules insert tuples into $V$. Consider the deletions. For each type of table reference, our theorems guarantee that the generated delete operations never delete tuples that should remain in $V$. Furthermore, these operations always delete all tuples that should no longer be in $V$. Consider the insertions. First, notice that all generated insert operations use nested select expressions based on the view definition itself. Since we know the view definition cannot produce duplicates, the set of tuples in insert operations never includes duplicates. Furthermore, our theorems (along with the "not in inserted V" clauses) guarantee that tuples already in $V$ are never inserted. Finally, in each case the insert operations produce all tuples that should be added to $V$.

We must consider that other production rules in addition to view-maintaining rules may be defined in the system. Although these rules cannot modify views, they can modify base tables. Our view-maintaining rules behave correctly even in the presence of other rules, and no additional rule ordering is necessary. Recall the semantics of rule execution (Section 3): a rule is considered with respect to the transition since the last time its action was executed; if its action has not yet been executed, it is considered with respect to the transition since the last rule assertion point (or start of the transaction). Hence, the first time a view-maintaining rule $R$ is triggered during rule processing, it processes all base table changes since the last assertion point. Suppose that, subsequently during rule processing, the base tables are changed by a non-view-maintaining rule. Then $R$ will be triggered again and will modify the view according to the new set of changes. When rule processing terminates, no rules are triggered, so all view-maintaining rules will have processed all relevant changes to base tables.

\section{Conclusions and Future Work}

We have described a facility that automatically derives a set of production rules to maintain a materialization of a user-defined view. This approach both frees the view definer from handling view maintenance and guarantees that the view remains correct. Through analysis techniques based on key information, incremental maintenance rules are generated whenever possible. Our facility allows the user to interact with the system: view definitions and key information can be modified to guarantee that the system produces efficient maintenance rules for frequent base table operations. In practice, efficient rules are possible for a wide class of views-efficiency relies on safe table references, and it can be seen from our criteria for safety that table references routinely fall into this class. In those cases where efficiency is not possible for the user's desired view, our system provides recognition of this fact; the user either may use the rules produced for automatic rematerialization or may decide that query modification is more appropriate.

We plan to implement our facility using the Starburst Rule System, then conduct experiments to evaluate the run-time efficiency of our approach on a variety of views. Meanwhile, we want to extend view analysis and rule generation so that the full power of SQL select statements can be used in view definitions. (We have started this and expect it to be tedious but not difficult.) Currently, the biggest drawback of our approach is that views with duplicates are not handled; we will consider ways to remove this restriction. We would like to add automatic rule optimization as a post-rule-generation component in our system. The rules produced by our 
method have a standard form, and in some cases can be optimized as in [CW90]. In addition, rules for different views could be merged and common subexpressions could be exploited as in [Han87). Finally, the properties guaranteed by our algorithms are useful in other areas (such as query optimization), and we intend to explore this connection.

\section{Acknowledgements}

Thanks to Guy Lohman and Laura Haas for helpful comments on an initial draft.

\section{References}

[ACL91] R. Agrawal, R.J, Cochrane, and B. Lindsay. On maintaining priorities in a production rule system. In Proceedings of the Seventeenth International Conference on Very Large Data Bases, Barcelona, Spain, September 1991.

[BLT86] J.A. Blakeley, P.-A. Larson, and F.W. Tompa. Efficiently updating materialized views. In Proceed. ings of the $A C M$ SIGMOD International Confer. ence on Management of Data, pages 61-71, Washington, D.C., June 1986.

[CG85] S. Ceri and G. Gottlob. Translating SQL into relational algebra: Optimization, semantics, and equivalence of SQL queries. IEEE Transactions on Software Engineering, 11(4):324-345, April 1985.

[CW90] S. Ceri and J. Widom. Deriving production rules for constraint maintenance. In Proceedings of the Sixteenth International Conference on Very Lorge Data Bases, pages 566-577, Brisbane, Australia, August 1990.

[CW91] S. Ceri and J. Widom. Deriving production rules for incremental view maintenance. IBM Research Report RJ 8027, IBM Almaden Research Center. March 1991.

[DE89] L.M.L. Delcambre and J.N. Etheredge, 'The Relational Production Language: A production language for relational databases. In L. Kerschberg. editor, Expert Database Systems-Proceedings from the Second International Conference, pages 333-351. Benjamin/Cummings, Redwood City, California, 1989.

[Han87] E. Hanson. Efficient Support for Rules and De. rived Objects in Relational Database Systems. PhD thesis, University of California, Berkeley, August 1987

[IBM88] IBM Form Number SC26-4348-1. IBM Systems Application Architecture, Common Programming Interface: Database Reference, October 1988.

[Kim82] W. Kim. On optimizing an SQL-like nested query. ACM Transactions on Database Systems 7(3):443-469, September 1982.

[KP81] S. Koenig and R. Paige. A transformational framework for the automatic control of derived data. In Proceedings of the Seventh International Conference on Very Large Data Bases, pages 306318, Cannes, France, September 1981.
[MD89] D.R. McCarthy and U. Dayal. The architecture of an active database management system. In Proceedings of the ACM SIGMOD International Conference on Management of Data, pages 215224, Portland, Oregon, May 1989.

[RC'BB89] A. Rosenthal, S. Chakravarthy, B. Blaustein, and J. Blakeley. Situation monitoring for active databases. In Proceedings of the Fifteenth International Conference on Very Large Data Bases, pages 455-464, Amsterdam, The Netherlands, August 1989.

[SI84] O. Shmueli and A. Itai. Maintenance of views. In Proceedings of the ACM SIGMOD International Conference on Management of Data, pages 240255, Boston, Massachusetts, May 1984.

[SJGP90] M. Stonebraker, A. Jhingran, J. Goh, and $S$. Potamianos. On rules, procedures, caching and views in data base systems. In Proceedings of the $A C M$ SIGMOD International Conference on Management of Data, pages 281-290, Atlantic City, New Jersey, May 1990.

SP89i A. Segev and J. Park. Updating distributed materialized views. IEEE Transactions on Knowledge and Data Engineering, 1(2):173-184, June 1989.

[Sto75] M. Stonebraker. Implementation of integrity constraints and views by query modification. In Proceedings of the ACM SIGMOD International Conference on Management of Data, pages 65-78, San Jose, California, May 1975.

[WCL9]; J. Widom, R.J. Cochrane, and B.G. Lindsay. Implementing set-oriented production rules as an extension to Starburst. In Proceedings of the Sev. enteenth International Conference on Very Large Data Bases, Barcelona, Spain, September 1991.

[WF90] J. Widom and S.J. Finkelstein. Set-oriented production rules in relational database systems. In Proceedings of the ACM SIGMOD International Conference on Management of Data, pages 259270. Atlantic City, New Jersey, May 1990.

'Wid91] J. Widom. Deduction in the Starburst production rule system. Submitted for publication, 1991. 